\title{
SHORT-RANGE WAKEFIELD IN A FLAT PILLBOX CAVITY GENERATED BY A SUB-RELATIVISTIC BEAM BUNCH
}

\author{
H. Wang ${ }^{*}$, R. Palmer, J. Gallardo, BNL, Upton, NY 11973, USA
}

\begin{abstract}
The short-range wakefield between two paralle conducting plates generated by a sub-relativistic beam bunch has been solved analytically by image charge method in time domain. Comparing with traditional modal analysis in frequency domain, this algorithm simplifies mathmatics and reveals great details of physics in electromagnetic field generation, propagation, reflection and causality. The calculated results have an excellent agreement with MAFIA and ABCI simulations in all range of beam velocities.
\end{abstract}

\section{INTRODUCTION}

Muon ionization cooling channel design for a neutrino factory [1] constitutes a sub-relativistic $(\beta=0.84)$ beam bunch passing through a beryllium-window pillbox cavity (7.82 $\mathrm{cm}$ gap, $14.28 \mathrm{~cm}$ radius). The beam dynamics simulation requires the space charge and wakefield effects to be included for the cooling rate calculation. There was a fundamental problem how to calculate the Coulomb and radiation fields when the bunch is still inside of cavity. In reality, the MAFIA in time domain [2] can calculate the wake potential. In tradition, the analytics use cavity eigen frequencies as a series expansion [3][4] to express the point charge wake and then integrate it into Gaussian bunch. Figure 1 shows their comparison. The agreement in long-range, after the bunch is outside of cavity, is very good. But in the short-range, even with 1600 modes in the analytics, the discrepancy is obvious. This problem can be understood in the following. The source of wakefield is beam bunch. After the bunch passing through the cavity, the ringing wake can be well dedicated by the cavity resonance modes. When the bunch is still inside of cavity, the wakefield will be dominated by the fields from the beam and their reflections in a few paths. To study the wakefield in the short range, an image charge method has been developed for a flat pillbox cavity, which is easy for a point charge to count Coulomb field and field reflections relative to the flat walls or mirrors. The reflection from the radial wall is far from the short-range.

\section{WAVES IN ONE-PLANE MODEL}

Electromagnetic fields generated by a $+q$ charge with a relative velocity $\beta=v / c$, moving out from an infinite large, perfect-conducting plane at $z=0$, can be deduced from Carron's note [5] in $(r, \phi, z)$ coordinates:

$$
e_{r}=\frac{q}{4 \pi \varepsilon_{0}}\left\{\frac{1}{\gamma^{2}}\left[\frac{r}{s_{-}^{3}}-\frac{r}{s_{+}^{3}}\right] u(c t-\mathfrak{R})+\frac{2 \beta r z}{\mathfrak{R}\left(\mathfrak{R}^{2}-\beta^{2} z^{2}\right)} \delta(c t-\mathfrak{R})\right\}
$$

*Current e-mail: haipeng@jlab.org

$$
\begin{aligned}
& e_{z}=\frac{q}{4 \pi \varepsilon_{0}}\left\{\frac{1}{\gamma^{2}}\left[\frac{z-\beta c t}{s_{-}{ }^{3}}-\frac{z+\beta c t}{s_{+}{ }^{3}}\right] u(c t-\Re)\right. \\
& \left.-\frac{2 \beta r^{2}}{\Re\left(\Re^{2}-\beta^{2} z^{2}\right)} \delta(c t-\Re)\right\} \\
& b_{\phi}=\frac{q}{4 \pi \varepsilon_{0} c}\left\{\frac{\beta}{\gamma^{2}}\left[\frac{r}{s_{-}^{3}}+\frac{r}{s_{+}^{3}}\right] u(c t-\Re)+\frac{2 \beta r}{\mathfrak{R}^{2}-\beta^{2} z^{2}} \delta(c t-\mathfrak{R})\right\}
\end{aligned}
$$

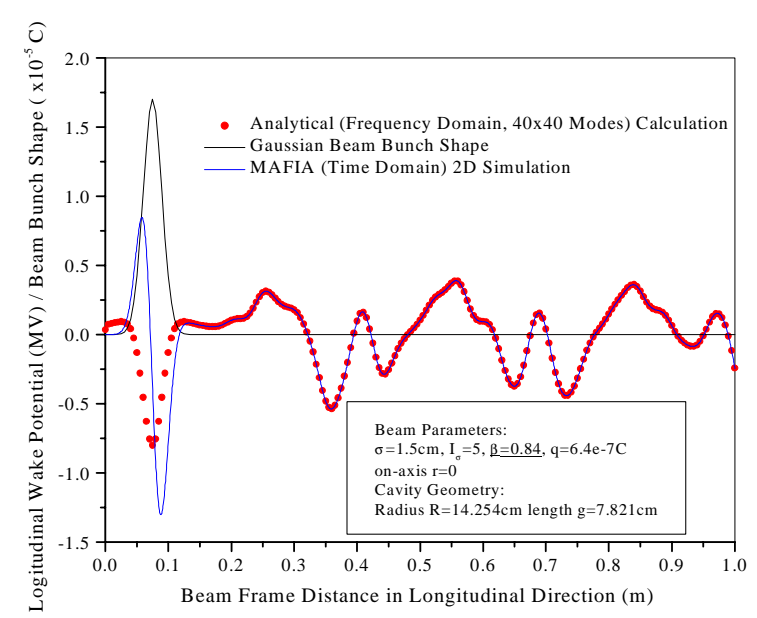

Figure 1: Long-range wake potential from a MAFIA calculation and a modal analysis.

Here $\quad s_{-} \equiv \sqrt{(\beta c t-z)^{2}+r^{2} / \gamma^{2}}, \quad s_{+} \equiv \sqrt{(\beta c t+z)^{2}+r^{2} / \gamma^{2}} \quad$ and $\Re \equiv \sqrt{r^{2}+z^{2}}$. The $c$ is the speed of light. $\gamma=1 / \sqrt{1-\beta^{2}}$.

All fields are independent of $\phi$. Each field component consists of three terms. Two terms with the step function $\mathrm{u}(\mathrm{ct}-\mathfrak{R})$ represent Coulomb field generated by a $+q$ source charge moving in $+z$ direction and a $-q$ image charge moving in $-z$ direction. The term with the Delta function $\delta($ ct- $\Re)$ represents the radiation field. It only rides on the spherical wavefront. The wavefront originates at $(r, z)=(0$, 0 ), with a radius of $\mathrm{ct}$, and expends in the speed $\mathrm{c}$. The $+q$ charge moves behind the wavefront. Coulomb field surrounds the $+q$, propagates with it, and expands between the conducting plane and the wavefront. As the particle approaches in relativistic $(\beta=1)$, Coulomb field vanishes and only radiation field left. The point charge is just riding on the wavefront. As the particle stays in static $(\beta=0)$, the radiation and magnetic fields disappear. Only Coulomb field left, in the form of Coulomb law.

In principle, a charge particle could not move out or in a perfect conductor due to the image charge effect. In reality, the conductivity of plate is always finite. The charge particle moves with certain energy. If the conductor is a thin foil, an energized particle can always pass through the conductor. In the mathematics, there is a 
singularity at the interception points. We can avoid this problem by excluding these points in the field integration.

\section{WAVES IN TWO-PLANE MODEL}

Before the wavefront reaches the second conductor plane, there is no new physics from the one-plane model. After that, a two-plane model needs to be developed. We added three additional physics points into (1)-(3).

- A backward radiation when the source charge exiting out from the second plane.

- Multiple field reflections will be superimposed. The reflected waves can be represented by infinite numbers of image charge fields generated at same time but originated at different locations.

- There is no Coulomb field emitted after the source charge left the second plane.

Use $s_{i-} \equiv \sqrt{(v t-z-2 i g)^{2}+r^{2} / \gamma^{2}}$ to replace the $s_{\text {, }}$ $s_{i+} \equiv \sqrt{(v t+z+2 i g)^{2}+r^{2} / \gamma^{2}}$ to replace $s_{+}$. . Use both $R_{i+} \equiv \sqrt{r^{2}+(z+2 i g)^{2}}$ and $\Re_{i-} \equiv \sqrt{r^{2}+(z-2 i g-g)^{2}} \quad$ to replace $\Re$. The order $i=0,-1,+1,-2,+2 \ldots$ represents the wavefront appearance sequence between two conducting planes. The $g$ is the gap between two conducting plates. Use normalized parameters: $E_{r} \equiv \frac{4 \pi \varepsilon_{0} g^{2}}{q} e_{r}, \quad E_{z} \equiv \frac{4 \pi \varepsilon_{0} g^{2}}{q} e_{z}$, $B_{\phi} \equiv \frac{4 \pi \varepsilon_{0} g^{2} c}{q} b_{\varphi}, R \equiv \frac{r}{g}, Z \equiv \frac{z}{g}, c T \equiv \frac{c t}{g}, R_{i+} \equiv \frac{\Re_{i+}}{g}, R_{i-} \equiv \frac{\Re_{i-}}{g}$,

$S_{i-} \equiv \gamma \frac{S_{i-}}{g}$ and $S_{i+} \equiv \gamma \frac{S_{i+}}{g}$. We got new field expression:

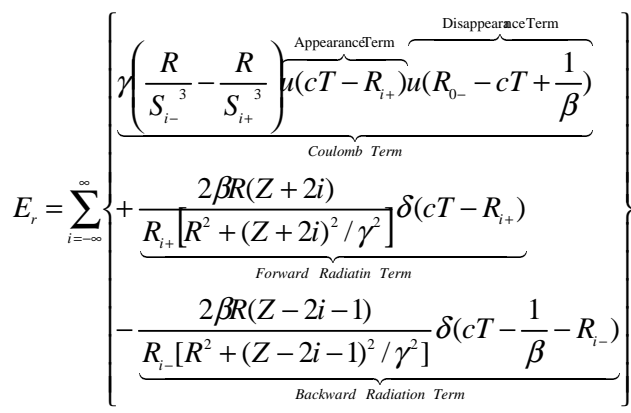

$E_{z}=\sum_{i=-\infty}^{\infty}\left\{\begin{array}{l}\gamma\left(\frac{Z+2 i-\beta c T}{S_{i-}{ }^{3}}-\frac{Z+2 i+\beta c T}{S_{i+}{ }^{3}}\right) u\left(c T-R_{i+}\right) \\ u\left(R_{0-}-c T+\frac{1}{\beta}\right) \\ -\frac{2 \beta R^{2}}{R_{i+}\left[R^{2}+(Z+2 i)^{2} / \gamma^{2}\right]} \delta\left(c T-R_{i+}\right) \\ +\frac{2 \beta R^{2}}{R_{i-}\left[R^{2}+(Z-2 i-1)^{2} / \gamma^{2}\right]} \delta\left(c T-\frac{1}{\beta}-R_{i-}\right)\end{array}\right\}$

$$
B_{\phi}=\sum_{i=-\infty}^{\infty}\left[\begin{array}{l}
\beta \gamma\left(\frac{R}{S_{i-}{ }^{3}}+\frac{R}{S_{i+}{ }^{3}}\right) u\left(c T-R_{i+}\right) u\left(R_{0-}-c T+\frac{1}{\beta}\right) \\
+\frac{2 \beta R}{R^{2}+(Z+2 i)^{2} / \gamma^{2}} \delta\left(c T-R_{i+}\right) \\
-\frac{2 \beta R}{R^{2}+(Z-2 i-1)^{2} / \gamma^{2}} \delta\left(c T-\frac{1}{\beta}-R_{i-}\right)
\end{array}\right]
$$

In equation (4), the term definition was braced out. A MathCAD program was written to show the electric field (4) and (5)'s snap shots in Figure 2.

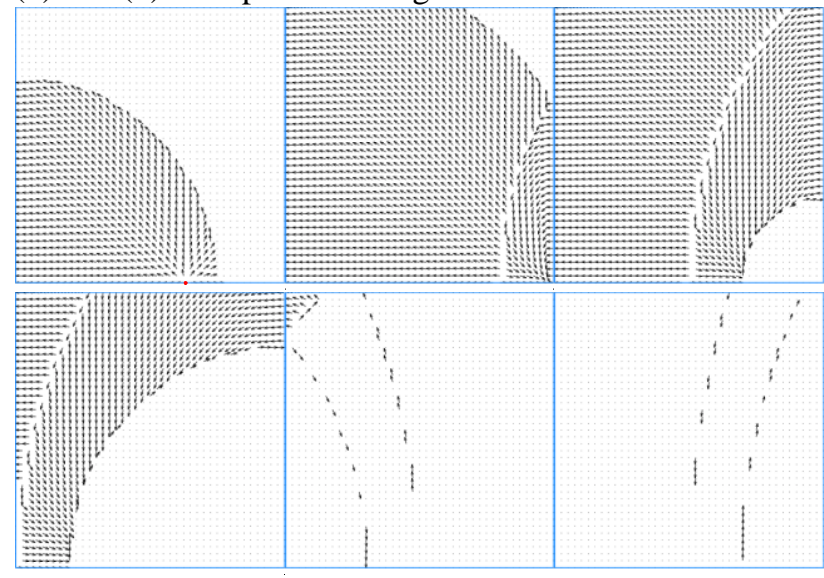

Figure 2: Six electric field vector plots at $\mathrm{T}=0.75 / \mathrm{c}, 1.2 / \mathrm{c}$, $1.5 / \mathrm{c}, 2.0 / \mathrm{c}, 2.5 / \mathrm{c}, 3.5 / \mathrm{c}$. In each time frame, the bottom line is beam axis in $\mathrm{Z}$, two sideboards are conducting planes in R. All arrow lengths normalize at each grid. Only arrow direction represents the field vector.

\section{POINT CHARGE WAKE POTENTIAL}

A witness charge behind or ahead of source charge could gain or loss energy due to the wakefield. The point charge wake potential can be used as a Green's function. Use the normalized parameters again: $W \equiv \frac{4 \pi \varepsilon_{0} g}{q} w, S \equiv \frac{s}{g}$.

The normalized longitudinal wake potential is:

$W_{\delta / I}(R, S)=\int_{0}^{1} E_{z}\left(Z, R, T=\frac{Z+S}{\beta c}\right) d Z$

The normalized transverse wake potential is:

$W_{\delta \perp}(R, S)=\int_{0}^{1}\left[E_{r}\left(Z, R, T=\frac{Z+S}{\beta c}\right)-\beta B_{\phi}\left(Z, R, T=\frac{Z+S}{\beta c}\right)\right] d Z$

The $+S$ is defined the distance of witness particle behind source particle.

\section{CAUSALITY ISSUE}

The equations (7) and (8) can be done analytically, but the algebra is very complicated due to causality issue. The causality is when the witness particle starts seeing the source particle's field, and when stops seeing the fields. That dictates by the integration limits. When converting the variable from time $\mathrm{T}$ to distance $\mathrm{Z}$, the integration limits will be re-confined by the step functions and the Delta functions. A math detail using "Z-S" plot was developed to identify these limits or the "open windows" seen by the witness particle [6]. The "window dimensions" depends on the field type, $\beta$ and $R$. Figure 3 shows one of the window examples. Table 1 summarizs these limits and conditions. The condition for "no window appears" means we only need a few terms to calculate the Coulomb field. Table 2 summaries the radiation wavefront positions. Again the "no radiation" condition gives the counts of terms for calculating radiation field. 


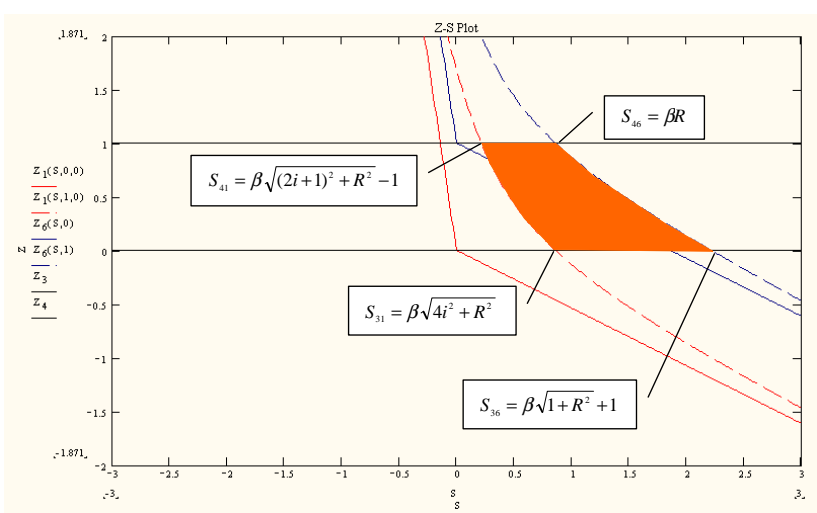

Figure 3: The "open window" in Z-S plot for $\beta=0.84, i=0$.

That is obvious advantage over the modal analysis. Another MathCAD program was developed [6]. It expands the integrations (7) and (8) analytically with the form of conditional branch functions in order to take account of the information in Tables 1 and 2.

Table 1: "Open window" dimensions of witness particle to see the source particle's Coulomb field in Z-S plot.

Here $Z_{1}=\gamma^{2}\left[\left(2 \beta^{2} i-S\right)+\beta \sqrt{(S-2 i)^{2}+\frac{R^{2}}{\gamma^{2}}}\right], \quad Z_{6}=1-S \gamma^{2}+\beta \gamma \sqrt{S^{2} \gamma^{2}+R^{2}}$ and $X$ is the root of equation $Z_{1}=Z_{6}$.

\begin{tabular}{|c|c|c|c|c|}
\hline$S_{\text {low }}$ & $S_{\text {high }}$ & $Z_{\text {low }}$ & $Z_{\text {high }}$ & conditions \\
\hline$\beta \sqrt{(2 i+1)^{2}+R^{2}}-1$ & $\beta R$ & $Z_{1}$ & 1 & \multirow{2}{*}{$\begin{array}{c}|2 i+1| \leq \frac{\sqrt{1+2 \beta R}}{\beta} \\
\text { and }\end{array}$} \\
\hline$\beta R$ & $\beta \sqrt{4 i^{2}+R^{2}}$ & $Z_{1}$ & $Z_{6}$ & \\
\hline$\beta \sqrt{4 i^{2}+R^{2}}$ & $\beta \sqrt{1+R^{2}}+1$ & 0 & $Z_{6}$ & $|A| \leq \frac{1}{2} \mid 1+\frac{1}{\beta^{2}}+\frac{2 \sqrt{1+R^{2}}}{\beta}$ \\
\hline$X$ & $\beta \sqrt{4 i^{2}+R^{2}}$ & $Z_{1}$ & $Z_{6}$ & \multirow{2}{*}{$\begin{array}{c}|2 i+1|>\frac{\sqrt{1+2 \beta R}}{\beta} \\
\text { and } \\
|i| \leq-\sqrt{1+\frac{1}{B}+\frac{2 \sqrt{1+R^{2}}}{B}}\end{array}$} \\
\hline$\beta \sqrt{4 i^{2}+R^{2}}$ & $\beta \sqrt{1+R^{2}}+1$ & 0 & $Z_{6}$ & \\
\hline \multicolumn{4}{|c|}{ No windows appears } & $|i|>\frac{1}{2} \sqrt{1+\frac{1}{\beta^{2}}+\frac{2 \sqrt{1+R^{2}}}{\beta}}$ \\
\hline
\end{tabular}

Table 2: Radiation wavefront positions. Here $Z_{1}=\gamma^{2}\left[\left(2 \beta^{2} i-S\right)+\beta \sqrt{(S-2 i)^{2}+\frac{R^{2}}{\gamma^{2}}}\right], \quad Z_{7}=-\gamma^{2}\left[S-\frac{1}{\gamma^{2}}+2 \beta^{2} i-\beta \sqrt{(S+2 i)^{2}+\frac{R^{2}}{\gamma^{2}}}\right] \cdot$

\begin{tabular}{|c|c|c|c|}
\hline$S_{\text {low }}$ & $S_{\text {hish }}$ & $Z_{\text {wauffout }}$ & note \\
\hline$\beta \sqrt{(2 i+1)^{2}+R^{2}}-1$ & $\beta \sqrt{4 i^{2}+R^{2}}$ & $Z_{1}$ & forward \\
\hline$\beta \sqrt{4 i^{2}+R^{2}}$ & $\beta \sqrt{(2 i+1)^{2}+R^{2}}$ & $Z_{7}$ & backward \\
\hline \multicolumn{2}{|r|}{$|i| \geq \frac{1}{2} \sqrt{\left(\frac{S+1}{\beta}\right)^{2}-R^{2}}-\frac{1}{2}$} & $\begin{array}{c}\text { no } \\
\text { radiation }\end{array}$ \\
\hline
\end{tabular}

\section{GAUSSIAN BEAM WAKE POTENTIAL}

Once the Green's function is known $W_{\delta}(\mathrm{s})$, for a Gaussian bunch, the normalized wake potential is:
$W\left(R, S^{\prime}\right)=\frac{1}{\sqrt{2 \pi}} \int_{-\infty}^{+\infty} \exp \left(-\frac{\left(S^{\prime}-S_{0}^{\prime}-S_{1}^{\prime}\right)^{2}}{2}\right) W_{\delta}\left(R, S_{1}^{\prime} \cdot \Sigma\right) d S_{1}^{\prime}$

Equation (9) applies both longitudinal and transverse. The parameters here are normalized. $S^{\prime} \equiv \frac{s}{\sigma}, \quad S_{0}^{\prime} \equiv \frac{s_{0}}{\sigma}$, $S_{1}^{\prime} \equiv \frac{s_{1}}{\sigma}, \Sigma \equiv \frac{\sigma}{g}$. The $s_{0}$ is the center of beam bunch. The $\sigma$ is the RMS bunch length. The integration (9) can be done numerically much faster by MathCAD with known analytical $W_{\delta}(R, S)$.

\section{CROSSCHECK WITH MAFIA}

The MAFIA wakefield simulation (both 2D and 3D in time domain) was first checked with code ABCI [7] (2D and $\beta=1$ beam) for a pillbox cavity. The agreement is very good both in short and long ranges. The ABCI also demonstrated the wave reflections similar to Figure 2. We then compared the MAFIA simulations with the calculations (9) in de-normalized form with a $\pm 5 \sigma$ bunch length. We tested in all $\beta$ and $r$ ranges. The agreement is excellent. The Figures 3 shows one of examples. The discrepancy happens only when $r$ is near the zero, since there is a singularity on axis. The MAFIA always avoids the $r=0$ case. In conclusion, this analytical approach reveals great physics details of short-range wakefield and benchmarks the MAFIA simulation in this region.

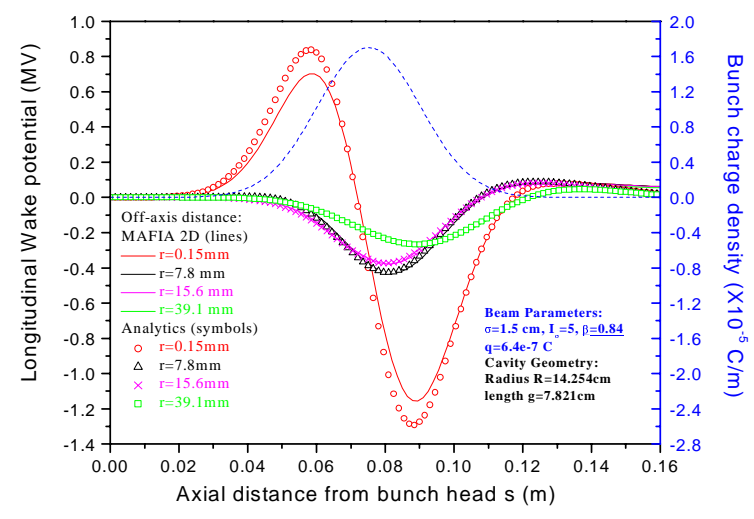

Figure 3: Longitudinal wake potentials from MAFIA and analytics for $\beta=0.84$ with different off-axis distances. The dot-line curve is the beam bunch shape.

\section{REFERENCES}

[1] http://www.cap.bnl.gov/mumu/studyii/FS2report.html.

[2] T. Weiland et al., http://www.cst.de/.

[3] T. Weiland, et al., "Wake Fields and Impedances", 1990 US-CERN Particle Accelerator School notes.

[4] A. Chao, P. Morton, SLAC Report PEP-105/SPEAR182 (Feb. 1975).

[5] N. J. Carron, "Fields of Particles and Beams Exiting a Conductor", A. J. Phys., submitted June 1998.

[6] H. Wang, http://wwwmucool.fnal.gov/mcnotes/muc0117.pdf.

[7] Y. Chin, CERN SL/94-02 (AP). 